\title{
Candidiasis en patología gineco-obstetricia
}

\author{
Raquel Lapides de Duplat*; Felisa Guarín Umaña**; Luis Vicente López Avila***
}

RESUMEN: Con el fin de establecer la prevalencia de candidiasis vaginal y las diferentes especies comprometidas en la infección cervicovaginal productora de leucorrea, se tomó una muestra representativa de la población que asiste a la consulta externa de ginecoobstetricia, en el Instituto Materno Infantil de Santafé de Bogotá durante el año de 1991 y los resultados fueron los siguientes:

1. La candidiasis vaginal representa un $26 \%$ de la muestra examinada (559 pacientes).

2. No se observa diferencia estadísticamente significativa en los diferentes grupos de edad.

3. Las especies de candida encontradas en orden de frecuencia fueron: C. albicans, C. stellatoidea, C. krussei, C. guillermondii, C.parapsilosis y C. pseudotropicalis.

4. En relación con el tipo de consulta se observó un marcado predominio de la candidiasis en la consulta prenatal (35\%), en relación con la ginecológica $(20 \%)$, diferencia estadísticamente significativa (p. 001).

5. En relación con la edad del embarazo y la frecuencia de candidiasis se observó que ésta aumenta a medida que avanza el embarazo (13\%) en el primer trimestre $y(44 \%)$ en el segundo.

6. Los factores predisponentes más frecuentes fueron en su orden: embarazo, diabetes, obesidad y uso de prostágenos.

7. En relación con la consulta ginecológica encontramos, que si bien es cierto, que C. albicans es la más frecuente, otras especies como stellatoidea y $k r u s s e i$ tienen también una alta frecuencia. $C$. krussei presenta una frecuencia seis veces mayor en consulta ginecológica en relación con la consulta prenatal.

PALABRAS CLAVES: Candidiasis vaginal.

SUMMARY: With the purpose of establishing the prevalence of vaginal Candidiasis and the different species involved in the cervicovaginal infection that produces leukorrhea, a sample representing the Gyneco-Obstetrics outpatient population of the Instituto Materno Infantil in Santafé de Bogotá, during 1991, and the results were the following:

1. Vaginal candidiasis represents $26 \%$ of the sample examined ( 559 patients).

2. No significantly statistical difference was observed in the different age groups.

3. The candida species found in order of frequency were: C. albicans, C. stellatoidea, C. krussei, C. guillermondii, C. parapsilosis, and C. pseudotropicalis.

4. In relation to the type of consultation a large predominance of candidiasis in pre-birth consultation (35\%), in relation to gynecologic consultation $(20 \%)$, a statistically significant difference (p.001).

5. In relation to pregnancy age and the frequency of candidiasis, it was noted that the latter increases with the pregnancy progress (13\%) in the first quarter and $(44 \%)$ in the second.

6. The most frequent incidental factors were in their order of significance: pregnancy, diabetes, obessity and use of prostagens.

7. In relation to the gynecologic consultation we found, that although it is true that C.albicans is the most frequently found, other species, such as stellatoidea and $k r u s s e i$ also are highly frequent. $C$. krussei shows a frequency 6 times larger in gynecologic consultation in relation to pre-birth consultation.

KEY WORDS: Vaginal candidiasis 


\section{Introducción}

La candida es una levadura oval que produce pseudomicelios en cultivos, tejidos y exudados. Sáprofito en la naturaleza y habitante normal en la flora intestinal en forma de levadura gemante (2). Se torna patógeno bajo circunstancias que favorecen su virulencia, como son desequilibrio de la flora normal por tratamiento prolongado con antibióticos; aumento de la glucosa en sangre y orina, como el caso de los diabéticos, el embarazo se considera como factor predisponente por el exceso de productos de tipo glucógeno en el epitelio vaginal; superficies de conductos húmedos y cálidos; el alcoholismo, obesidad, factores hormonales, factores que alteran los mecanismos de defensainmunológica, desnutrición, hemopatías, prematuridad y similares (16).

Particularmente la micosis vaginal está condicionada por otros factores además de los ya citados, como la administración de anticonceptivos, acidez vaginal e higiene deficiente. Este tipo de vaginitis se ha observado en niveles socioeconómicos bajos y también más a menudo durante el embarazo que en mujeres no grávidas (de 15 a $30 \%$ en el primercaso y de 7 al 16\% en el segundo) (8) (11) (17). Según estudios realizados recientemente (5) en poblaciones femeninas de 11 a 18 años con vulvovaginitis y leucorrea se encontró una frecuencia de candida albicans de $18 \%$ de los casos examinados. Puede suponerse que la frecuencia sería mayor si el estudio incluyera mujeres de más edad en período fértil y con vida sexual activa.

La candidiasis se presenta como una infección aguda o subaguda en la cual el hongo puede producir lesiones en la boca, vagina, piel, uñas, bronquios, pulmones y en algunas ocasiones llega a producir septicemia, endocarditis, meningitis, además de infecciones intestinales y del tracto urinario (4.11.17).

Dentro del género candida encontramos varias especies de las cuales unas son más patógenas que otras. La patogenicidad puede establecerse por medio de la antigenicidad, utilizando técnicas de inmunofluorescencia y aglutinación. Se ha encontrado el grado de antigenicidad de las diferentes especies estableciendo un título de $1 / 1280$ para albicans y stellatoidea y $1 / 160$ para krussei, tropicalis y pseudotropicalis. La frecuencia de las diferentes especies tienen el siguiente orden descendente: albicans, krussei, tropicalis y guillermondii.

Con el fin de mejorar el diagnóstico y orientar el manejo de la candidosis vaginal en nuestro medio, se realizó un estudio descriptivo de corte transversal, para establecer la prevalencia de la especie de hongos comprometidos en el cuadro clínico de las pacientes que asisten a la consulta externa de ginecología y obstetricia del Instituto Materno Infantil de Santafé de Bogotá y que han consultado por flujo genital o que durante un examen genital se descubrió infección del cervix, vagina o vulva.

* Profesora Asociada Unidad de Micología Dpto. de Microbiología. Facultad de Medicina Universidad Nacional de Colombia.

** Profesora Asociada Unidad de Micología Dpto. de Microbiología. Facultad de Medicina Universidad Nacional de Colombia.

*** Profesor Asociado Dpto. de Ginecobstetricia Facultad de Medicina Instituto Materno Infantil. Universidad Nacional de Colombia.

\section{Materiales y métodos}

Para seleccionar una muestra representativa de la población que asiste a esta consulta, se tomó una muestra probabilística, sin etapas, aleatoria simple sin restitución del universo. Para tal fin se llevó a cabo el siguiente procedimiento: Teniendo en cuenta que en la consulta externa de ginecología y obstetricia del IMI se atienden aproximadamente 700 consultas al mes, para un año serían 8400 . De otra parte, se ha señalado que la frecuencia de candida puede representar un $18 \%$ de los casos de vulvovaginitis y leucorrea. Pero esta frecuencia podría llegar a un $30 \%$ si se toma una población con riesgo aumentado, ya sea por las condiciones socioeconómicas bajas, el embarazo, el uso de anticonceptivos y antibióticos. Con base en estos datos puede estimarse la muestra requerida en la siguiente forma:

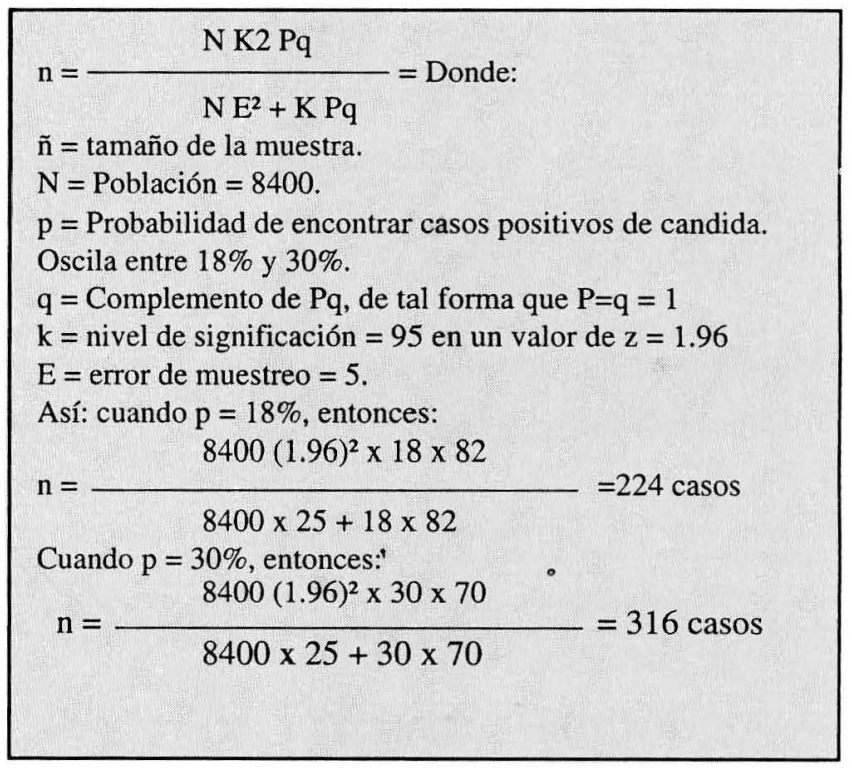

En consecuencia, la muestra a utilizar tendrá un nivel mínimo de 350 casos.

Durante el período de estudio se tomaron 559 muestras de igual número de pacientes con cérvico vaginitis y leucorrea. Las muestras se tomaron de tres sitios diferentes; introito (con ayuda de espéculo), de formix posterior y exocervix, con un escobillón, el cual es sumergido en un tubo con solución salina isotónica estéril y así se transportó al laboratorio de Micología de la Facultad de Medicina de la Universidad Nacional donde se procesó en la siguiente forma:

a) coloración de Gram para la identificación de levaduras.

b) cultivo en medio de Sabouraud para identificar candidosis.

c) cultivo en lactosa, glucosa, sacarosa, maltosa y galactosa para identificar especie.

Para la Candida albicans se usó suero humano.

\section{Resultados}

De las 559 muestras tomadas, se encontraron 144 casos positivas para candida, esto es un $26 \%$ y 415 casos negativos $74 \%$. La muestra estuvo conformada por 336 casos ginecológicas y 223 obstétricas prenatales.

Se analizaron las diferentes variables y los resultados fueron los siguientes: 
Tabla 1

SINTOMATOLOGIA REFERIDA

\begin{tabular}{|lcc|}
\hline \multirow{2}{*}{ Síntomas } & \multicolumn{2}{c|}{ Frecuencia } \\
& No. & $\%$ \\
\hline Leucorrea & 144 & 100 \\
Prurito genital & 139 & 95 \\
Mal olor & 121 & 84 \\
Ardor genital & 107 & 75 \\
\hline
\end{tabular}

Como se puede apreciar los pacientes referían síntomas muy frecuentes y muy molestos.

Se estudiaron las características organolépticas de la secreción y los hallazgos fueron los siguientes:

Tabla 2

CARACTERISTICAS ORGANOLEPTICAS

\begin{tabular}{|c|c|c|c|c|}
\hline Características & No. $\%$ & No. $\%$ & No. $\%$ & No. $\%$ \\
\hline \multirow[t]{2}{*}{ Aspecto } & Mucoso & Grumoso & Acuoso & Espumoso \\
\hline & 8660 & 4934 & 2.7 & $5 \quad 3.3$ \\
\hline \multirow[t]{2}{*}{$\mathrm{Ph}$ Vaginal } & 4.0 & 5.0 & 6.0 & 7.0 \\
\hline & $\begin{array}{ll}1 & 0.7\end{array}$ & $85 \quad 60$ & $15 \quad 10.3$ & $42 \quad 29$ \\
\hline \multirow[t]{2}{*}{ Cantidad } & Abundante & Moderado & Escaso & \\
\hline & $84 \quad 58$ & $21 \quad 15$ & $39 \quad 27$ & \\
\hline \multirow[t]{2}{*}{ Color } & Amarillo & Blanco & & \\
\hline & $30 \quad 21$ & $114 \quad 79$ & & \\
\hline
\end{tabular}

Se investigó la frecuencia por grupos de edad no hallándose diferencia estadísticamente significativa en los diferentes grupos como se puede ver en la tabla siguiente:

Tabla 3

DISTRIBUCION POR EDADES

\begin{tabular}{|ccc|}
\hline Grupos de edad & No. de casos & $\%$ \\
\hline $15-20$ & 26 & 18 \\
$21-25$ & 25 & 17 \\
$26-30$ & 18 & 13 \\
$31-35$ & 18 & 13 \\
$36-40$ & 28 & 19 \\
$41-$ & 29 & 20 \\
\hline
\end{tabular}
mos:

En relación con las diferentes especies identificadas tene-
Tabla 4

ESPECIES DE CANDIDA IDENTIFICADAS

\begin{tabular}{|lcc|}
\hline Especie & No. de casos & $\%$ \\
\hline C. albicans & 68 & 47.2 \\
C. stelletoidea & 44 & 30.5 \\
C. krussei & 14 & 9.7 \\
C. guillermondii & 11 & 4.6 \\
C. parapsilosis & 6 & 4.6 \\
C. pseudotropicales & 1 & 0.7 \\
\hline
\end{tabular}

Como se puede observar en la tabla anterior más de la mitad de la candidiasis corresponden a otras especies diferentes a albicans.

En relación con los factores de riesgo identificados se obtuvieron los siguientes resultados:

Tabla 5

\section{FACTORES DE RIESGO MAS FRECUENTES}

\begin{tabular}{|llll|}
\hline Factores & $\begin{array}{l}\text { No. de casos } \\
\text { Encontrados }\end{array}$ & $(+)$ & $(\%)$ \\
\hline Diabetes & 100 & 26 & 26 \\
Obesidad & 68 & 17 & 25 \\
Anticonceptivos & 25 & 5 & 20 \\
\hline
\end{tabular}

El comportamiento en los diferentes factores fue muy semejante y no hubo diferencia estadísticamente significativa entre ellos ni con la muestra general, solamente se observó un aumento en el embarazo, estadísticamente significativo (P .05)

Se estudiaron las frecuencias en relación con el tipo de consulta ginecológica u obstétrica y la especie de candida presente, encontrándose los siguientes datos:

Tabla 6

TIPO DE CONSULTA Y ESPECIE DE CANDIDA

\begin{tabular}{|lcccc|}
\hline Especie & C. Ginecológica & $\%$ & C. Obstétrica & \multicolumn{1}{l|}{$\%$} \\
\hline C. albicans & 28 & 19.40 & 40 & 27.70 \\
C. stelletoidea & 18 & 12.50 & 26 & 18.00 \\
C. krussei & 12 & 8.33 & 2 & 1.38 \\
C. guillermondii & 6 & 4.16 & 5 & 3.47 \\
C. parapsilosis & 3 & 2.08 & 3 & 2.08 \\
C. pseudotropicalis & 0 & 0.00 & 1 & 0.60 \\
Total & 67 & 47.60 & 77 & 53.40 \\
\hline
\end{tabular}

En términos generales la distribución fue proporcional al total en ambos grupos, exceptuando al grupo $C$. krussei que fue mayor en la consulta ginecológica.

Se estudió la candidiasis en relación con la edad del embarazo y los resultados fueron los siguientes según la especie: 
Tabla 7

FRECUENCIA DE CANDIDIASIS SEGUN TRIMESTRE DE EMBARAZO

\begin{tabular}{|lcccc|}
\hline Especie & $103 \mathrm{~m}$. & $203 \mathrm{~m}$. & $303 \mathrm{~m}$ & Total \\
C. albicans & 4 & 16 & 20 & 40 \\
C. stellatoidea & 4 & 11 & 11 & 26 \\
C. krussei & 0 & 2 & 0 & 2 \\
C. guillermondii & 1 & 3 & 1 & 5 \\
C. pseudotropicales & 1 & 0 & 0 & 1 \\
C. parapsilosis & 0 & 2 & 1 & 3 \\
Total & 10 & 34 & 33 & 77 \\
\hline
\end{tabular}

Como se puede ver en la tabla anterior la frecuencia se manifiesta en forma progresiva según la edad del embarazo, en general en todas las especies, lo cual nos sugiere el posible riesgo que implica el embarazo en la génesis de la candidiasis genital en nuestro medio.

Se estudió la frecuencia de las diferentes especies en la consulta ginecológica y la distribución fue la siguiente:

Tabla 8

DISTRIBUCION DE LA CANDIDIASIS EN LA CONSULTA GINECOLOGICA

\begin{tabular}{|lcrcc|}
\hline Especie & No. de casos & \multicolumn{1}{c|}{$\%$} & \% por casos + \% en muestras \\
\hline C. albicans & 28 & 42 & 19.4 & 5.0 \\
C. stelletoidea & 18 & 27 & 12.5 & 3.2 \\
C. krussei & 12 & 18 & 8.3 & 2.1 \\
C. guillermondii & 6 & 9 & 4.2 & 1.1 \\
C. parapsilosis & 3 & 4 & 2.1 & 0.6 \\
C. pseudotropicalis & 0 & 0 & 0.0 & 0.0 \\
Total & 67 & 100 & 46.5 & 12.0 \\
\hline
\end{tabular}

Como podemos ver en la tabla anterior la candidiasis más frecuente es la albicans, sin embargo, los demás grupos suman más de la mitad en total, lo cual nos hace pensar en el valor que puedan tener, al menos en cuanto a frecuencia se refiere otras especies fuera de la C. albicans en la candidiosis genital.

\section{Discusión}

En la muestra de 559 pacientes de los que asisten a la consulta externa del I.M.I. por prestar Cérvico - Vaginitis y flujo genital, durante el año 1991, se encontró un 26\% de casos positivos para candidiasis genital.

Frecuencia ésta acorde con estudios realizados en otras latitudes, las que señalan una frecuencia de 7 a $30 \%$ en la población Gineco - Obstétrica que consulta por flujo genital.

En relación con la población obstétrica $34.52 \%$ y con la ginecológica $20 \%$.

Los síntomas más frecuentemente referidos por las pacientes fueron secreción genital 144 casos en $100 \%$, prurito genital 137 casos, un 955, mal olor 121, un 184\%, 107 casos para un $75 \%$ de ardor genital. Sintomatología esta hallada por Da Costa (1969).
Analizadas las características organolépticas de la secreción se encontraron los siguientes resultados: aspecto mucoso $34 \%$; espumoso un $3.3 \%$ y acuoso un $2.7 \%$. Frecuencias éstas halladas por otros autores. Cuando se estudió el color de la secreción se identificó el blanco como el más frecuente con un $79 \%$ y el amarillo un $21 \%$.

En relación con la cantidad fue abundante en un $58 \%$, moderado en un $15 \%$ y escaso en un $27 \%$.

El ph vaginal se presentó en la siguiente forma: (ph 4.0), un $10.5 \%$ y (ph de 7.0 o más) un $29 \%$. Datos estos que concuerdan con los obtenidos por Calatroni (1988) (Tabla 2).

Se estudió la frecuencia por grupos de edad no hallándose diferencia alguna (Tabla 3 ).

En relación con las especies identificadas tenemos que el comportamiento fue el siguiente:

Candida albicans $47.2 \%$, la más frecuente; stellatoidea un $30.5 \%$, krussei un $9.7 \%$, guillermondii un $7.6 \%$, parapsilosis un 4.55 y pseudotropicalis $0.7 \%$. Datos en desacuerdo con Hurley (1981), quien dice que además de candida albicans y glabrata, no tiene importancia ninguna otra especie de candida. Como se puede ver, en la tabla 4, más de la mitad de candidas son de otra especie, sobre todo, en relación con stellatoidea, krussei y guillermondii con frecuencias no despreciables.

Cuando se analiza la frecuencia de candida según los factores de riesgo, tenemos que la Diabetes se encuentra en primer lugar con $20 \%$, la obesidad; le sigue con $12 \%$, los anticonceptivos con $3.4 \%$ y los antibióticos con $1.3 \%$, en la muestra examinada, factores estos que en alguna forma han colaborado en la presencia de la candidiasis, no se observó predominio de especie alguna en relación con el factor.

En relación con el tipo de consulta se observó un predominio de candidiasis en la consulta prenatal con 77 casos positivos en una población de 223 embarazadas con flujo genital, esto es, una frecuencia de $35 \%$, comparado con la consulta ginecológica con 67 casos positivos en una población de 336 pacientes no embarazadas con flujo genital, un $20 \%$. Diferencia estadísticamente significativa (p.001).

Analizando la especie de candidiasis comprometida se encontró una distribución proporcional excepto en el caso de la C. krussei la cual fue 6 veces más frecuente en la consulta ginecológica que en la obstétrica.

En relación con la frecuencia de candidiasis y edad de embarazo se observó un aumento de frecuencia a medida que avanza la edad del embarazo, así: durante el primer trimestre 10 casos (13\%), en el segundo trimestre del embarazo 34 casos (44\%) y en el tercer trimestre 33 casos (43\%). Lo cual nos confirma una vez más que el embarazo aumenta el riesgo de candidiasis vaginal (16), exceptuando C. krussei la cual presentó su mayor frecuencia en la mujer embarazada.

Se analizaron las diferentes especies en relación con la consulta ginecológica y los datos fueron los siguientes: si bien es cierto que $C$. albicans tiene la mayor frecuencia 28 casos $(41 \%)$ de la muestra positiva en una población de 336 pacientes con flujo genital, C. stellatoidea con 18 casos (27\%) y C. krussei 12 casos para ( $18 \%$ ) de casos positivos, no son frecuencias despreciables y se deben tener en cuenta en la consulta de clínica ginecológica. 


\section{BIBLIOGRAFIA}

1. Baily and Scotts; Diagnostic Microbiology 7 th edition. The C.V. Company, St. Louis, Toronto, Princeton, 1986.

2. Beneke ES and A. Rogers. Medical Micology Laboratory y Manual 4 th ed. Minneapolis, Minn. Burgees Publishing, Companay, 1980.

3. Cardonas de O. Dora., Raad A. Jorge. Servicio Seccional de Salud. Universidad Católica. Manizales, Colombia. Etiología de las enfermedades de transmisión sexual en Manizales.

4. Corriere JN. Jr. Pathologic Changes in the human urinary tract due to anovulatory drug administration. J. Surg. Oncol. 1973; 5: 167.

5. Gottlieb Beatriz, et al. Vulvovaginitis infecciosa en adolescentes. Estudio Parasitológico, bacterilógico y micológico. Parasitol al día. 1987; 11: 4-7. Chile.

6. Jones JM. Laboratory diagnosis of invasiva candidiasis. Clin. Microbiol. Rev. 1990; 3: 32-45.

7. Kominsky D. and L. Quinlan A. Simple inexpensive method for rapid identification of candida albicans. Am y Clin Path 1963; 40: 143.

8. Kass, E.H. Hormones and host resistance to infection Bacteriol Rev, 1960; 24: 177.
9. Landau J.W., N. Dabrowa and V.D. Newcomer. The rapid formation in serum of filaments by candida albicans and Invest. Derm. 1965; 44: 172.

10. Ordóñez Nelly, et al. Las pruebas inmunológicas en el diagnóstico micológico. Instituto Nacional de Salud. Bogotá, Colombia. 1987.

11. Restrepo I. Marcos. Estudio de 1.200 flujos vaginales, Cit. Antioquia. Médica. Feb. 1964. Vol, 4 No. 1 pp 4-26.

12. Rhoads y L. wright C. Redfuld R. Chronical Vaginal Candidiasis in woman with human inmunodeficiency virus infection. Jama 1987; 257: 3105-3107.

13. Romano, A.H. Ainswoth y Sussamans. The fungi. 1966. Vol. II Cap. 7; Press N.Y.

14. Rose Noel, Friedman Herman. Manual of Clinical Inmunology. American Society for Microbiology. Washington D.C. 1976.

15. Soto Jaime; Examen Directo: una ayuda diagnóstica Hospital Militar Central; Bogotá, Colombia. 1987.

16. Vowkennles, J. Sympositum Ciba. Ag. 1956; Tomo 4 No. 3, Bélgica.

17. Whittle, H. Candida infection of skin and mucosae P. 3, Antwerp Belgium-infection. Jama 1987; 257: 3105-3107. 